\title{
Impacto de las actitudes emprendedoras en los estudiantes de la universidad de Guayaquil carrera comunicación social
}

\section{Impact of entrepreneurial attitudes in students of the university of Guayaquil career social communication}

Teresa Mirian Santamaría López

Francisco Bravo Villagómez

Byron Castro Villacis

Universidad de Guayaquil, Ecuador

Autor para correspondencia: teresa.santamarial@ug.edu.ec, francisco_gabriel84@hotmail.com, bycavi2009@hotmail.com

Fecha de recepción: 24 de Noviembre de 2016 - Fecha de aceptación: 10 de Enero de 2017

\section{Resumen}

El artículo se trata de un producto de investigación en donde se analiza las actitudes emprendedoras de los estudiantes y su evolución desde que ingresan a la universidad hasta dos años de experiencia académica. Se ha utilizado de manera estratégica preguntas que invitan a resolver inquietudes elementales para la resolución de una problemática que se viene dando hace muchos años en nuestro medio como lo es la desmotivación o su disminución de la misma a la hora de emprender mientras van pasando los años de estudio universitario. Se consideran las actitudes de asunción al riesgo, control interno percibido, evaluación de la situación económica, necesidad de logro, autoestima e innovación. Estas variables inciden de una u otra forma en la toma de una decisión final de los emprendedores, que en ocasiones desisten de sus proyectos. El Resultado que se obtiene es la disminución de las actitudes emprendedoras y se proponen diferentes acciones.

Palabras clave: Actitudes; emprendedores; educación; motivación y emprendimiento

\begin{abstract}
The article is a research product that analyzes the entrepreneurial attitudes of students and their evolution from entering university to two years of academic experience. It has been strategically used questions that invite us to solve elementary concerns for the resolution of a problem that has been occurring for many years in our environment as it is the demotivation or its decrease of the same when it comes to undertaking as the years go by of university study. Attitudes of risk assumption, perceived internal control, evaluation of the economic situation, need for achievement, self-esteem and innovation are considered. These variables affect one way or another in making a final decision of the entrepreneurs, who sometimes give up their projects. The result is the reduction of entrepreneurial attitudes and different actions are proposed.
\end{abstract}

Keywords: Attitudes; entrepreneurs; education; motivation and entrepreneurship 


\section{Introducción}

En esta investigación se buscó conocer cuáles eran las actitudes emprendedoras de los estudiantes de la Universidad de Guayaquil (UG) su evolución. Se analizó la figura del emprendedor desde diferentes ópticas, y especialmente según las actitudes emprendedoras definidas como: necesidad de logro, autoestima, innovación, control percibido interno, evaluación de la situación económica, y asunción de riesgo.

El estudio empírico se basó en la investigación realizada por la Dra. Ibáñez (2001) en España, específicamente, en el País Vasco. De esa investigación se extrajeron los cuestionarios para ser aplicados a los estudiantes que ingresaban a la UCO. El mismo se volvió a pasar al segundo año y al tercer año de permanencia en la UG, a fin de conocer la evolución de las actitudes emprendedoras de los estudiantes. Finalmente se realizó entrevistas en profundidad para conocer las razones de la variación de sus actitudes y se propusieron diferentes formas de incentivar el emprendimiento en la universidad.

\section{Importancia del tema}

Diferentes autores y organismos internacionales han reconocido la importancia de los emprendedores a la hora de considerar el desarrollo económico de los distintos países, entendiendo al emprendedor como una persona que percibe una oportunidad y crea un nuevo negocio con ella como lo indican Bygrave y Hofer (1991).

El hecho de desarrollar la capacidad emprendedora como forma de combatir el desempleo es bueno, pero el efecto no es inmediato, ya que puede demorarse una década en ver el resultado (Audretsch y Keilbach, 2005). En este sentido, Angelelli y Prats (2005) explican que el impacto de la actividad emprendedora es positivo sobre el crecimiento y el empleo, siempre que se lo considere en un horizonte de mediano y largo plazo (Isbenberg, 2010).

Los emprendedores están transformando el mundo (Timmons y Spinelli, 2007). Ellos crean trabajo a partir de la innovación, reconocen oportunidades, aplican capital de riesgo y cambian la economía. Utilizan el cambio como una oportunidad de mejora económica, no como un problema. Desde esta perspectiva, un emprendedor puede ser un gerente profesional o un colaborador cualquiera de la empresa. Se necesita de la experimentación, de la imaginación, de la innovación para detectar oportunidades, haciendo que el emprendedor sea un factor clave en la empresa y en la sociedad, sobre la base del «saber y aprender a emprender» (Bueno, 2003). Así, deben manejarse tres directrices fundamentales:

$\checkmark$ Aprender a aprender, que combina las actitudes (visiones y valores) con el conocimiento (explícito);

$\checkmark$ Aprender a hacer, que combina las actitudes con las capacidades entendidas como habilidades y destrezas que se relacionan con el talento;

$\checkmark$ Aprender a emprender, que combina los conocimientos con las capacidades.

La tendencia actual en gestión, destinada a que las empresas sobrevivan a la globalización y a la cada vez mayor competencia, es la promoción de una cultura emprendedora dentro de la 
empresa, apoyada por gerentes emprendedores que marquen pautas (Bhardwa, Sushil y Momaya, 2007).

\section{Objetivo de la investigación}

La educación tiene una responsabilidad central en identificar y fomentar a los que pueden ser emprendedores (Hatten, 1996). Por esta razón, el ideal buscado es desarrollar actitudes emprendedoras en los estudiantes de forma que un número significativo de graduados cree su propia empresa, se desarrolle como emprendedor y de esta forma contribuya al desarrollo del país y a la generación de empleos.

Para analizar las actitudes emprendedoras de los estudiantes la investigación se basó en la tesis doctoral de Ibáñez (2001), que analizó las actitudes emprendedoras de los estudiantes de la CAPV (Comunidad Autónoma del País Vasco) utilizando la escala EAO (Entrepreneurial Attitude Orientation Scale) desarrollada por Robinson (1987). En este caso, el estudio se centra únicamente en los estudiantes de la Universidad de Guayaquil.

El objetivo general fue investigar las actitudes emprendedoras de los estudiantes de la UG y los objetivos específicos fueron:

- Conocer si existen diferencias en las actitudes y en las distintas opiniones que tienen los estudiantes de la UG sobre la creación de empresas en función de las carreras que cursan.

- Determinar la relación entre la actividad emprendedora de los padres con las actitudes emprendedoras de los estudiantes.

- Conocer la relación entre los estudiantes que trabajan y sus actitudes emprendedoras.

- Investigar la evolución de las actitudes emprendedoras al cabo de un año de permanencia en la UG

- Analizar la evolución de las actitudes al cabo de dos años de permanencia en UG (dos años desde su ingreso).

- Identificar la actitud emprendedora de los estudiantes de la Licenciatura en Dirección de Empresas.

- Realizar una propuesta de formación.

\section{Marco teórico}

La base para estudiar las actitudes emprendedoras de los estudiantes se basó en los cuatro factores definidos por Robinson y partir de los cuales se creó la escala EAO y pueden ser explicados de la siguiente manera:

- Necesidad de logro en los negocios, referida a resultados concretos con la creación y desarrollo de nuevos emprendimientos.

- Innovación en los negocios, percibida como el funcionamiento de una empresa en una forma nueva y única.

- Control percibido interno en los negocios, como forma de influir en los resultados empresariales. 
- Autoestima relacionada con los negocios, como confianza y sentimiento de competencia para llevar acabo el negocio.

La necesidad de logro (McClelland, 1961) se puede definir como una característica básica de los emprendedores, que actúan de una determinada forma:

- Necesitan ser responsables de sus actos, tener libertad para actuar y control de la situación, además de identificarse con el éxito cuando lo logran;

- Buscan retroalimentación continua sobre sus actos para luego modificar sus objetivos, y así lograr el éxito;

- Fijan metas que sean retadoras pero a la vez realistas y alcanzables;

- No les agradan las tareas rutinarias sino las que el esfuerzo influya en sus resultados;

- A la hora de escoger compañeros de trabajo, prefieren expertos en vez de amigos. Al analizar la innovación es importante distinguir entre emprendedor e inventor. El inventor tiende a tener una educación elevada, está dispuesto a enfrentar riesgos y tolera bien la ambigüedad y la incertidumbre. Su medida de realización es el número de inventos desarrollados y de patentes propias, demostrando poco interés en el beneficio económico. En cambio, un emprendedor está interesado en la organización de un nuevo negocio y se compromete con su supervivencia. El inventor está enamorado con su invención y se resiste a que le hagan modificaciones para su comercialización (Mueller y Heidemann, 2002).

Por su propia naturaleza, la innovación está impregnada de incertidumbre y lleva al riesgo como factor impulsor o limitador en el análisis y decisión que realiza el agente innovador. Las decisiones tomadas bajo condiciones de incertidumbre tienden a enfrentar riesgos mayores que resultan en errores o fracasos. Aunque exista la posibilidad de revertir esos procesos, estos riesgos pueden causar elevadas pérdidas a las empresas (Brandão y Piñeiro, 2004).

Tener una actitud emprendedora exige un nuevo espíritu, una nueva mentalidad que hace que el hombre y la mujer no esperen, sino que actúen, que se involucren en la toma de sus propias decisiones (Profesor Sérvulo Anzola, Director del programa emprendedor del ITESMMéxico).

Otra de las actitudes del emprendedor es la asunción de riesgo. El riesgo posee tres componentes: el tamaño de la pérdida, la probabilidad de ocurrencia y la vulnerabilidad de esta.

Además, según Ibáñez (2001) se reconocen tres fuentes que determinan el riesgo:

- Insuficiencia de control sobre personas, recursos, el tiempo y la información.

- Limitación de información, ya sea por no ser completa o no ser fiable.

- Escasez de tiempo cuando se debe tomar una decisión sin tener que poseer la información completa.

El emprendedor es una persona que asume riesgos. Del mismo modo, una persona con mayor tolerancia al riesgo tendrá una mayor asunción de riesgo y, por lo tanto, un menor grado de aversión a este. Ello implicará que la persona tendrá menos miedo en auto emplearse y en 
crear su propio emprendimiento. Los individuos con una alta necesidad de logro tienen una propensión al riesgo moderada; por tanto, parece que la necesidad de logro afecta a la percepción del riesgo y el emprendedor asume riesgos en forma calculada (Bealey, 2000).

El control percibido interno o bien el locus of control es el elemento que muestra el lugar donde la persona sitúa el control de su vida. El control percibido es interno si la persona cree que los resultados que obtiene en su vida son debidos a sí mismo. En cambio, una persona con control percibido externo creerá que su actuación no va a influir en los resultados que obtenga. Basará la explicación de los acontecimientos en la suerte, el azar, la influencia de otras personas o grupos sociales de poder (Ayerbe y Buenetxea, 2000). Por lo tanto, dicha actitud es fundamental para el trabajo y para una actividad emprendedora.

El control percibido interno se relaciona positivamente con el esfuerzo en el trabajo, con nuevas ideas, con iniciativa, etcétera, y negativamente con la creencia de la suerte, ideas viejas, decepción ante la no consecución de metas, obediencia, etc. Una persona con control percibido interno es más persuasiva, busca más información, es más propensa a olvidar los recuerdos del fracaso. Es muy importante que el emprendedor posea esta actitud, ya que a lo largo del emprendizaje tendrá fracasos que deberá afrontar.

Efectivamente, la persona -y su empresa- tienen más éxito si posee un elevado control interno, y responsabilidad. Esto hace que el emprendedor seleccione cuidadosamente su plan de negocios, haciendo más probable el resultado exitoso (Blackman, 2004).

La autoestima, se puede explicar cómo la evaluación que se hace de sí mismo. Es la confianza en la propia capacidad para pensar y afrontar los desafíos que surgen todos los días. La autoestima positiva hace que las personas busquen objetivos exigentes que la estimulen y, al lograrlos, su autoestima aumenta aún más (Branden, 1993).

La autoestima es una cualidad importante para que una persona sea capaz de enfrentar muchos obstáculos y convertirse en emprendedora. Cuanto más confíe en sus propias actitudes, con mayor perseverancia buscará sus objetivos y se convencerá de que es competente para lograrlo.

La autoestima necesita tiempo y trabajo para lograrse; no basta solamente con pedirla. Los individuos que la poseen son los que tienden a valorar sus logros y habilidades en forma objetiva, sin negarlos ni exagerarlos. Asimismo las personas con baja autoestima son vulnerables y se vuelven un blanco fácil de atacar. La autoestima actúa como un moderador frente a los factores de riesgo del trabajo, razón por la cual es tan importante que el emprendedor posea una alta autoestima, capaz de resguardar la armonía y la dignidad en condiciones de estrés (Harvey y Keashly, 2003).

Por otra parte, Korunka et al. (2003), en sus investigaciones encuentran tres razones diferentes que hacen que la persona emprenda:

- A pesar de su voluntad, por ejemplo, al perder el trabajo (o bien cuando deja su trabajo porque no encuentra cabida para sus ideas) 
- Porque lo desea, ya que encontró una oportunidad.

- Porque tiene el apoyo de una red.

Una de las razones principales es que emprenden porque han encontrado una oportunidad, definida como cualquier actividad que requiere invertir escasos recursos a la espera de un retorno y sienten que son capaces de aprovechar esa ocasión (Austin et al., 2006).

\section{Formación de emprendedores}

Dada que la investigación se realiza en estudiantes universitarios, es fundamental analizar las diferentes formas en que se pueden formar los emprendedores, aprovechándolas oportunidades que detectaron. La formación de emprendedores debe darse tanto en carreras de grado, maestría y doctorado, uniendo la realidad y los negocios con la educación (Thursby et al., 2009).

Según Henry et al. (2005), antes de comenzar a analizar qué se debe enseñar que hay que distinguir entre:

1. educación sobre emprendimiento

2. educación para emprender

3. educación en la empresa

En el primer caso, el objetivo se basa en enseñar a los estudiantes diferentes aspectos sobre la creación de empresas, sobre las actitudes y los valores propios de empezar una empresa, gerencial y hacerla crecer. La enseñanza es desde una perspectiva teórica. Los forman para que en un futuro deseen crear su propia empresa.

En el segundo caso, la óptica es diferente y se trata de formar emprendedores que creen sus propias empresas. Se les enseña a preparar su propio plan de negocios. Con respecto a la educación en la empresa, se da en emprendedores que ya crearon su empresa y desean hacerla crecer. Los que toman esos cursos desean actualizar sus conocimientos.

En cuanto a lo que se debe enseñar para ser emprendedores, Hisrich, Peters, (2002), consideran que los emprendedores deben tener:

a) Habilidades técnicas tales como saber comunicarse en forma escrita y oral, conocimientos y habilidades en gestión y organización.

b) Habilidades de administración de empresas tales como planificar, tomar decisiones, saber comercializar y llevar la contabilidad.

c) Habilidades personales tales como control percibido interno, innovación, asunción de riesgo, perseverancia y liderazgo.

En concordancia con lo anterior, se sugiere que la educación en emprendimiento debe abarcar la parte cognitiva (información, habilidades), afectiva (emoción, temperamento), y comportamental (motivaciones o deseos) indicados por Koiranen y Ruohotie (2001). 
Diferentes estudios han mostrado la eficacia de la educación en la formación de emprendedores (Drost, 2010).

Existe autores que consideran que lo esencial es enseñar a realizar planes de negocios ya que, por más sencillos que sean, guían la actividad del emprendedor y los motiva para luego emprender (Honig, 2004).

Los planes de negocios han surgido desde 1980 como una necesidad a partir de la información solicitada por bancos y contadores. Son importantes para las relaciones con los stakeholders, para facilitar la comunicación y la negociación, pero no son la clave del éxito de los emprendedores, a pesar de que se enseñe en las escuelas de negocios (Gibb, 2005).

El problema real es que los estudiantes no toman seriamente el plan de negocios realizado dentro del aula, lo que lleva a que puedan fracasar en sus proyectos (Neck y Brush, 2011).

A su vez se ha reconocido la importancia de realizar competencia de planes de negocios como forma de incentivar a los estudiantes que emprendan (Russell et al., 2008).

Con respecto a la búsqueda de oportunidades, puede ser enseñada en el aula y que se obtienen buenos resultados. Uno de los puntos primordiales es saber registrar cuándo aparece la idea y luego desarrollarla (De Tienne y Chandler, 2004).

Existen las diferentes formas en que se puede enseñar a emprender como son: haciendo planes de negocios, poniendo en marcha una empresa, realizando consultorías a emprendedores, a través de juegos de simulación en computadoras, por medio de simulación de comportamientos, entrevistando emprendedores, viviendo casos, utilizando video y filmaciones (Kuratko, 2005). Reconociéndose a su vez lo resultados que estas actividades brindan la formación de los emprendedores (Kumara y Sahasranam, 2009).

Los investigadores deben conocer cómo los individuos aprenden y cómo se puede influir en su aprendizaje para fortalecer la enseñanza del emprendimiento. Del mismo modo, hay que resaltar la importancia de los conocimientos y de la creatividad al momento de aceptar las diferencias individuales y de formar emprendedores (Corbett, 2005).

Hoy en día sigue vigente la teoría de Piaget para la enseñanza del emprendimiento, en la cual la persona aprende mejor después de un desequilibrio. Esta teoría se reafirma con los que consideran que la mejor forma de aprender esa través de las emociones y de superar los fracasos.

Una forma de hacerlo es escribir diarios o entrevistar a personas que han fracasado y que manifiesten cómo superaron esa vivencia. Se trata de que el alumno viva el fracaso como real y lo intente superar. La crítica que se le hace a este modelo es que, dando tanta importancia al hecho de vencer el fracaso, se puede desalentar a los estudiantes a que inicien una nueva actividad o negocio (Shepherd, 2004). Por tanto, para la formación de emprendedores es fundamental quelleven a la práctica un emprendimiento más que aprender en forma teórica (Eldelman et al., 2008). 
A su vez, Neck y Green (2011), reconocen que para formar emprendedores se necesita una metodología que depende delas personas pero no del tipo de persona y que para enseñar sobre emprendimiento se requiere práctica.

\section{Metodología}

El diseño del cuestionario, Ibáñez (2001) se inspiró en los trabajos realizados por De Dios y Ayerbe. La parte central del cuestionario está basada en el Entre preneurial Attitude Orientation (EAO) Scale, desarrollado en Estados Unidospor Robinson (1987). Ver anexo 1.

Se analizó a los mismos estudiantes durante tres años para conocer su evolución (estudio longitudinal) así como los estudiantes que estaban en el mismo año en diferentes carreras (estudio transversal). Los estudiantes que pertenecían carreras empresariales (EE) eran los de: Dirección de empresas, Negocios internacionales, Economía, Contador público y Relaciones Laborales. Los de carreras no empresariales (EnoE) eran: Ingeniería, Comunicación, Derecho, Psicología. En total se aplicaron 886 cuestionarios.

Las metodologías empleadas fueron: análisis factorial, diferencias de las medias poblacionales, análisis de varianza, análisis de componentes principales y análisis de correspondencias múltiples. Como forma de obtener una medida más objetiva, se analizó la puntuación total obtenida en el test (Anexo 1), llamada «actitud emprendedora global» (AEG).

Este cálculo se realizó haciendo la suma de los enunciados que englobaban a los cuatro factores: asunción al riesgo, motivación al logro, control interno percibido y autoestima.

\section{Conclusiones}

En base a los resultados de la investigación se concluye que la única forma en que se puede desarrollar el emprendimiento es aprender haciendo y aprender emprendiendo. Para formar emprendedores hay que incrementar las actitudes emprendedoras, desarrollar la inteligencia emocional, y educar por competencias.

Los resultados obtenidos muestran que se debe realizar mayor énfasis en emprendimiento para los estudiantes dela licenciatura en Dirección de Empresas compuesta por asignaturas obligatorias como: Desarrollo de Habilidades emprendedoras, Empresas Familiares, Creatividad e Innovación, Emprendimiento y asignaturas opcionales como: Finanzas Internacionales, Marketing Internacional, Negocios internacionales. Las características de estas asignaturas es su carácter internacional, ya que para los estudiantes del Ecuador, es fundamental su visión hacia el mundo exterior ya que el mercado interno es muy pequeño.

Otra manera de fomentar el emprendimiento entre los estudiantes es por medio de un concurso de ideas y planes de negocios. El mismo ya ha venido desarrollándose y permite que estudiantes de grado y postgrado así como de diferentes carreras se integren y creen un proyecto en forma conjunta. La ventaja que trae aparejada la integración es que logran complementarse y apoyarse, requisito fundamental para hacer que un emprendimiento funcione. Otra forma en que 
se apoya y fomenta el emprendimiento, es por medio de una Pre-incubadora llamada Nexo que asigna una tutora los estudiantes que lo requieran para apoyarlos a hacer un plan de negocios.

En un futuro cercano, se tratará de brindar a los estudiantes, como indica Kailer (2009) mayor apoyo en la parte financiera, una red de contactos con clientes y proveedores, y ayudas con respeto a patentes y licencias.

Asimismo, a través del Programa de Desarrollo Emprendedor, se ha creado una línea de investigación que se ofrece a diferentes estudiantes e investigadores. El Programa está unido a diferentes redes como: Programa Emprender, Redde Relapi de Infodev, Bizspark de Microsoft, Red Urunova,Red Latinoamericana de Emprendimiento de Cladea.

Lo interesante de esta investigación, fue haber detectado la necesidad de fomentar el espíritu emprendedor en los estudiantes de la UG y comenzar a implementar los cambios a nivel de determinadas carreras, así como de la Facultad de Comunicación Social de la Universidad de Guayaquil, contribuyendo de esta forma al desarrollo y crecimiento del país.

\section{Bibliografía}

Angelelli, P.; Prats, J. (2005). Fomento de la actividad emprendedora en América Latina y el Caribe. Serie de Informes Técnicos del Departamento de Desarrollo Sostenible. Banco Interamericano de Desarrollo.

Austin, J; Stevenson H. y Wei-Skillern, J. (2006). «Social and commercial entrepreneurship: same, different, or both?».Entreprenuership Theory and Practice.30 (1), pp. 1-22.

Ayerbe, M. y Buenetxea, E. (2000). La cultura del trabajo yla actitud emprendedora en el ámbito profesional de la C.A.P.V. Cuadernos Sociológicos Vascos. Vitoria-Gasteiz: Servicio Central de Publicaciones del Gobierno Vasco.

Begley, K. (2000). Learning styles among a select group of entrepreneurs: Implications for entrepreneurship programs at institutions of higher education in the United States. Tesis de Doctorado para la obtención del título de Doctor en Educación en Innovación y Liderazgo. Delaware, Estados Unidos: Wilmington.

Bhardwaj, B; Sushil y Momaya, K. (2007). «Corporate entrepreneurship: application of moderator method». Singapore Management Review. 29(1), pp. 47-58.

Blackman, A. (2004). Entrepreneurs: Interrelationships between their characteristics, values, expectations, management practices and SME performance. Australian Digital Theses Program.

Griffith University.

Brandão, M. y Pinheiro, D. (2004). «Programa de empreendedorismo eminstituições de ensino superior: Reflexões a partir de algunas experiencias canadienses y americanas». 
Branden, N. (1993). El poder de la autoestima. Cómo potenciar este importante recurso psicológico. Buenos Aires: Paidós.

Bueno, E. (2003). «Emprender en la sociedad del conocimiento: el capital del aprendizaje como dinamizador del capital intelectual». En Arnal Losilla (coord.). Creación de empresa. Los mejores textos, Barcelona: Ariel, pp. 61-80.

Neck, H, y Greene, P. (2011). «Entrepreneurship Education:Known Worlds and New Frontiers».Journal of Small Business Management. 49(1), pp. 55-70.

Revista de Negocios, Centro de Ciencias Sociales Aplicadas, Universidad Regional de Blumenau, Brasil, 9(2), pp. 117-126.

Robinson, P. (1987). Prediction of entrepreneurship based onan attitude consistency model. Tesis doctoral. Provo, Utah: University of Brigham Young...

Russell, R, Atchison, M. y Brooks R. (2008). «Business plan competitions in tertiary institutions: encouraging entrepreneurship education». Journal of Higher Education Policy \&Management.30 (2).pp 123-138.

Shepherd, D. (2004). «Educating Entrepreneurship students about emotion and learning from failure». Academy of Management Learning and Education, 3(3), pp. 274-287.

Timmons, J.; Spinelli, S. (2007).New venture creation: entrepreneurship for the 21 st century. $7 .^{\text {a }}$ ed. Nueva York: McGraw-Hill.

Washington, DC. Disponible en: 〈http://www.iadb.org/sds/doc/int1b2.pdf〉Audretsch, D. y Keilbach, M. (2005).

«Entrepreneurship capital and regional growth».Annals of Regional Science.39 (3), pp. 457-469. 